\title{
Global State-Feedback Control for Switched Nonlinear Time-Delay Systems via Dynamic Gains
}

\author{
Hui Ye $\mathbb{D}^{1,2}$ Bin Jiang $\mathbb{D}^{1},{ }^{1}$ Hao Yang, ${ }^{1}$ and Gui-Hua Zhao ${ }^{2}$ \\ ${ }^{1}$ College of Automation Engineering, Nanjing University of Aeronautics \& Astronautics, Nanjing 210016, China \\ ${ }^{2}$ School of Science, Jiangsu University of Science and Technology, Zhenjiang 212000, China \\ Correspondence should be addressed to Bin Jiang; binjiang@nuaa.edu.cn
}

Received 23 August 2019; Revised 19 November 2019; Accepted 27 November 2019; Published 14 February 2020

Academic Editor: Mauro Gaggero

Copyright $\odot 2020$ Hui Ye et al. This is an open access article distributed under the Creative Commons Attribution License, which permits unrestricted use, distribution, and reproduction in any medium, provided the original work is properly cited.

In this paper, the problem of global state-feedback control is investigated for a class of switched nonlinear time-delay systems. In order to obtain a less-conservative common dynamic gain update law across subsystems, we construct different dynamic gain update laws for individual subsystems. Based on multiple Lyapunov function approach and adding one power integrator technique, the delay-independent controllers for all subsystems and a proper switching law are designed to guarantee that the states of the switched nonlinear time-delay systems can be globally asymptotically to the origin; meanwhile, all the signals of the closed-loop system are bounded. Finally, an example is provided to demonstrate the effectiveness of the proposed method.

\section{Introduction}

A switched system is a branch of hybrid system. It consists of multiple subsystems that are either continuous-time or discrete-time ones and a switching law that defines specific subsystems active at instants of time. Switched nonlinear systems are commonly used in practice, such as robot control systems, networked control systems, and aircraft control systems [1-5]. In recent years, the design of switching strategies as well as stability and stabilization issues are critical in control theory and engineering and have achieved considerable results ([6-17] and the references therein). To address these issues, many methods have been presented, such as the common Lyapunov function, the single Lyapunov function method, the average dwell time scheme, and the multiple Lyapunov function method and switched Lyapunov functions. In particular, multiple Lyapunov function method along with the selection of appropriate switching signals provides an effective way for stability analysis and stabilization of switched nonlinear systems [18].

As is well known, time-delays are experienced from time to time in practical control systems, such as ecological systems and industrial procedures, which may cause system instability (see $[19,20]$ and the references therein). For nonswitched nonlinear systems, high-order nonlinear systems without time-delays have been thoroughly investigated in [21]. In the case of growth constraints, the problem of output feedback control was considered in $[22,23]$ for highorder time-delay systems.

Motivated by [23-26], we construct a memoryless statefeedback controller and abolish the upper bound of the timedelay. For each subsystem, a dynamic gain is introduced in the procedure of the recursive design. With the dynamic gains introduced, some other negative terms are found in the derivative of the Lyapunov function, which may offset stronger system nonlinearities. Although prior knowledge of the upper bound of the time-delay is not required, the system can be regulated to its origin, while all the closedloop signals are bounded. The main contributions of this paper are summarized as follows: (i) without any growth condition on the time-delay systems nonlinearities, delayindependent, nonsmooth but $C^{0}$ state-feedback controllers with dynamic gains are developed, which regulate the states of the time-delay system, while keeping the boundedness of the closed-loop system. (ii) The issue of global stabilization 
for switched nonlinear time-delay systems is studied for the first time by combining multiple Lyapunov function, adding one power integrator method and dynamic gains technique.

Notations. Throughout this paper, $\mathbb{R}^{n}$ denotes the $n$-dimensional real space; $\mathbb{R}_{+}$refers to the set of all nonnegative real numbers; $\mathscr{C}^{i}$ denotes the set of all functions with continuous $i$ th partial derivatives; and $\bar{l}_{i}=\left(l_{1}, \ldots, l_{i}\right)^{T}$.

\section{Preliminaries and Problem Statement}

In this paper, we consider the problem of the global statefeedback control for switched nonlinear time-delay system described by

$$
\begin{aligned}
& \dot{x}_{i}(t)=x_{i+1}^{p_{i \sigma(t)}}(t)+f_{i \sigma(t)}\left(\bar{x}_{i}(t), \bar{x}_{i}(t-d)\right) \\
& +g_{i \sigma(t)}(x(t)), \quad(i=1, \ldots, n-1), \\
& \dot{x}_{n}(t)=u_{\sigma(t)}^{p_{n \sigma(t)}}(t)+f_{n \sigma(t)}(x(t), x(t-d)) \text {, } \\
& x(s)=\zeta(s), \quad s \in[-d, 0],
\end{aligned}
$$

where $x=\left(x_{1}, \ldots, x_{n}\right)^{T} \in \mathbb{R}^{n}$ is the system state. For $i=$ $1, \ldots, n, \bar{x}_{i}=\left(x_{1}, \ldots, x_{i}\right)^{T}$ and $\bar{x}_{i}(t-d)=\left(x_{1}(t-d)\right.$, $\left.\ldots, x_{i}(t-d)\right)^{T} . d \geq 0$ is an unknown system time-delay. $\sigma(t)$ is a piecewise switching signal taking its values in a finite set $M=\{1, \ldots, m\}, m$ being the number of subsystems. For $i=1, \ldots, n, k \in M, p_{i k}$ are positive odd integers, $u_{k} \in \mathbb{R}$ is the control input of the $k$ th subsystem. $f_{i k}: \mathbb{R}^{2 i} \longrightarrow \mathbb{R}$ and $g_{i k}: \mathbb{R}^{n} \longrightarrow \mathbb{R}$, are $\mathscr{C}^{1}$ functions with $f_{i k}(0,0)=0$ and $g_{i k}(0)=0 . \zeta(s) \in \mathbb{R}^{n}$ is a continuous function, and takes its values in $[-d, 0]$. Moreover, it is assumed that the state of system (1) will not jump at the moment of switching, i.e., the trajectory $x(t)$ is a continuous one.

To address the state-feedback control problem of the switched nonlinear time-delay systems (1), the following useful lemmas are given which will be used throughout the paper.

Lemma 1 (see [26]). For positive real numbers $p$ and $q$, there exists a positive real-valued function $\varrho(x, y)$ such that

$$
|x|^{p}|y|^{q} \leq \frac{p}{p+q} \varrho(x, y)|x|^{p+q}+\frac{q}{p+q} \varrho(x, y)^{-(p / q)}|y|^{p+q} .
$$

Lemma 2 (see [21]). For $x, y \in \mathbb{R}$ and an integer $m \geq 1$, the following inequality holds

$$
\begin{gathered}
|x+y|^{m} \leq 2^{m-1}\left|x^{m}+y^{m}\right|, \\
(|x|+|y|)^{1 / m} \leq|x|^{1 / m}+|y|^{1 / m} \leq 2^{(m-1) / m}(|x|+|y|)^{1 / m} .
\end{gathered}
$$

If $m$ is a positive odd, then

$$
|x-y|^{m} \leq 2^{m-1}\left|x^{m}-y^{m}\right|
$$

Lemma 3 (see [25]). For a continuous function $f(x, y)$, there are $\mathscr{C}^{\infty}$ functions $a(x) \geq 0, b(y) \geq 0, c(x) \geq 1$ and $d(x) \geq 1$, such that $|f(x, y)| \leq a(x)+b(y), \quad|f(x, y)| \leq$ $c(x) d(y)$.

In this paper, we firstly consider the switched nonlinear time-delay systems described by

$$
\begin{aligned}
\dot{x}_{i}(t) & =x_{i+1}^{p_{i \sigma(t)}}(t)+f_{i \sigma(t)}\left(\bar{x}_{i}(t), \bar{x}_{i}(t-d)\right), \quad(i=1, \ldots, n-1), \\
\dot{x}_{n}(t) & =u_{\sigma(t)}^{p_{n \sigma(t)}}(t)+f_{n \sigma(t)}(x(t), x(t-d)), \\
x(s) & =\zeta(s), \quad s \in[-d, 0]
\end{aligned}
$$

which is a special case of system (1) with $g_{i \sigma(t)}(x)=0$. Then, we will design a set of controllers for all subsystems and a switching law to implement global regulation of system (1).

Since $f_{i k}(\cdot)$ is $\mathscr{C}^{1}$ and $f_{i k}(0,0)=0$, it can be obtained from Lemma 3 that there exist smooth functions $\varphi_{i k j}\left(\bar{x}_{i}\right)$ and $\psi_{i k j}\left(\bar{x}_{i}(t-d)\right), i=1, \ldots, n, k \in M, j=1, \ldots, i$, such that

$$
\left|f_{i k}(\cdot)\right| \leq \sum_{j=1}^{i} \varphi_{i k j}\left(\bar{x}_{j}\right)\left|x_{j}\right|+\sum_{j=1}^{i} \psi_{i k j}\left(\bar{x}_{j}(t-d)\right)\left|x_{j}(t-d)\right| .
$$

\section{Controller Design}

Based on the idea of multiple Lyapunov function and adding one power integrator technique, the controller design procedure is summarized as follows.

Step 1. Let $\xi_{1 k}=x_{1}$ and choose the Lyapunov function $\widetilde{V}_{1 k}\left(x_{1}, l_{1}\right)=(1 / 2)\left(1+\left(1 / l_{1}\right)\right) \xi_{1 k}^{2}$ with $l_{1}(t) \geq 1$ being a dynamic gain to be determined in the next step. By simple calculation, the time derivative of $\widetilde{V}_{1 k}$ yields

$$
\begin{aligned}
\dot{\tilde{V}}_{1 k}\left(x_{1}, l_{1}\right) & =\left(1+\frac{1}{l_{1}}\right) \xi_{1 k}\left(x_{2}^{p_{1 k}}+f_{1 k}\right)-\frac{i_{1}}{2 l_{1}^{2}} \xi_{1 k}^{2} \\
& \leq\left(1+\frac{1}{l_{1}}\right) \xi_{1 k} x_{2 k}^{* p_{1 k}}+2\left|\xi_{1 k} \xi_{2 k}\right|+2\left|\xi_{1 k} f_{1 k}\right|-\frac{i_{1}}{2 l_{1}^{2}} \xi_{1 k}^{2},
\end{aligned}
$$

where $x_{2 k}^{* p_{1 k}}$ is the first virtual controller to be design later and $\xi_{2 k}=x_{2}^{p_{1 k}}-x_{2 k}^{* p_{1 k}}$.

It can be obtained from (6) that there exist $\mathscr{C}^{\infty}$ functions $\varphi_{1 k 1}\left(x_{1}\right)$ and $\psi_{1 k 1}\left(x_{1}(t-d)\right)$ such that

$$
\left|f_{1 k}\right| \leq \varphi_{1 k 1}\left(x_{1}\right)\left|x_{1}\right|+\psi_{1 k 1}\left(x_{1}(t-d)\right)\left|x_{1}(t-d)\right| .
$$

Consequently, one has

$$
2\left|\xi_{1 k} f_{1 k}\right| \leq 2 \xi_{1 k}^{2} \varphi_{1 k 1}\left(x_{1}\right)+\xi_{1 k}^{2}+\xi_{1 k}^{2}(t-d) \psi_{1 k 1}^{2}\left(x_{1}(t-d)\right) .
$$

Consider the Lyapunov-Krasovskii functional

$$
V_{1 k}=\tilde{V}_{1 k}+\int_{t-d}^{t} \xi_{1 k}^{2}(\mu) \psi_{1 k 1}^{2}(x(\mu)) \mathrm{d} \mu,
$$

with (7) and (9) in mind, the time derivative of $V_{1 k}$ becomes 


$$
\begin{aligned}
\dot{V}_{1 k} \leq & \left(1+\frac{1}{l_{1}}\right) \xi_{1 k} x_{2 k}^{* p_{1 k}}+\xi_{2 k}^{2}-\frac{i_{1}}{2 l_{1}^{2}} \xi_{1 k}^{2}+\xi_{1 k}^{2}\left(2+2 \varphi_{1 k 1}\left(x_{1}\right)\right. \\
& \left.+\psi_{1 k 1}^{2}\left(x_{1}\right)\right) .
\end{aligned}
$$

Design the virtual controller as

$$
\begin{aligned}
x_{2 k}^{* p_{1 k}} & =-\xi_{1 k}\left(\frac{n-1}{2}+3+2 \varphi_{1 k 1}\left(x_{1}\right)+\psi_{1 k 1}^{2}\left(x_{1}\right)+\alpha_{1 k}\left(x_{1}\right)\right) \\
& \triangleq-\xi_{1 k} \beta_{1 k}\left(x_{1}\right),
\end{aligned}
$$

where $\alpha_{1 k}\left(x_{1}\right), \beta_{1 k}\left(x_{1}\right) \in \mathscr{C}^{\infty}$. This together with $l_{1}(t) \geq 1$ leads to

$$
\dot{V}_{1 k} \leq-\left(1+\alpha_{1 k}\left(x_{1}\right)\right) \xi_{1 k}^{2}-\frac{n-1}{2} \xi_{1 k}^{2}+\xi_{2 k}^{2}-\frac{i_{1}}{2 l_{1}^{2}} \xi_{1 k}^{2} .
$$

Step 2. Take the Lyapunov-Krasovskii functional into consideration

$$
\tilde{V}_{2 k}=V_{1 k}+\frac{1}{l_{1}}\left(1+\frac{1}{l_{2}}\right) W_{2 k}+\frac{1}{2 l_{1} l_{2}} \xi_{1 k}^{2},
$$

where $W_{2 k}=\int_{x_{2 k}^{*}}^{x_{2}}\left(\mu^{p_{1 k}}-x_{2 k}^{* p_{1 k}}\right)^{2-1 / p_{1 k}} \mathrm{~d} \mu$ and $l_{2}(t) \geq 1$ is a dynamic gain to be determined later. Then, one has

$$
\begin{aligned}
& \frac{\partial W_{2 k}}{\partial x_{2}}=\xi_{2 k}^{2-\left(1 / p_{1 k}\right)}, \\
& \frac{\partial W_{2 k}}{\partial x_{1}}=\left(\frac{1}{p_{1 k}}-2\right) \frac{\partial x_{2 k}^{* p_{1 k}}}{\partial x_{1}} \int_{x_{2 k}^{*}}^{x_{2}}\left(\mu^{p_{1 k}}-x_{2 k}^{* p_{1 k}}\right)^{1-\left(1 / p_{1 k}\right)} \mathrm{d} \mu, \\
& \lambda_{2 k}\left(x_{2}-x_{2 k}^{*}\right)^{2 p_{1 k}} \leq W_{2 k}\left(x_{1}, x_{2}\right) \leq\left(2^{p_{1 k}}-1\right) \xi_{2 k}^{2},
\end{aligned}
$$

where $\lambda_{2 k}>0$ is a constant for $k \in M$.

Due to $l_{j}(t) \geq 1, j=1,2$, combining (13)-(15), one has

$$
\begin{aligned}
\dot{\widetilde{V}}_{2 k} \leq & -\left(1+\alpha_{1 k}\left(x_{1}\right)\right) \xi_{1 k}^{2}-\frac{n-1}{2} \xi_{1 k}^{2}+\xi_{2 k}^{2}-\frac{i_{1}}{2 l_{1}^{2}} \xi_{1 k}^{2} \\
& +\frac{1}{l_{1}}\left(1+\frac{1}{l_{2}}\right) \xi_{2 k}^{2-\left(1 / p_{1 k}\right)}\left(x_{3 k}^{* p_{2 k}}+x_{3}^{p_{2 k}}-x_{3 k}^{* p_{2 k}}\right) \\
& +\frac{2}{l_{1}}\left|\xi_{2 k}^{2-\left(1 / p_{1 k}\right)} f_{2 k}+\frac{\partial W_{2 k}}{\partial x_{1}} \dot{x}_{1}\right|+\frac{1}{l_{1} l_{2}} \xi_{1 k} \dot{x}_{1} \\
& -\frac{i_{1}}{l_{1}^{2}} W_{2 k}-\frac{i_{1} l_{2}+l_{1} \dot{l}_{2}}{l_{1}^{2} l_{2}^{2}}\left(W_{2 k}+\frac{\xi_{1 k}^{2}}{2}\right),
\end{aligned}
$$

where $x_{3 k}^{* p_{2 k}}$ is the second virtual controller.

From (6), (12), (15), and Lemmas 1 and 2, one has

$$
\begin{aligned}
\frac{2}{l_{1}}\left|\xi_{2 k}^{2-\left(1 / p_{1 k}\right)} f_{2 k}\right| \leq & \xi_{2 k}^{2} \Psi_{2 k, 1}\left(\bar{x}_{2}\right)+\frac{1}{l_{1}} \xi_{1 k}^{2}(t-d) \Psi_{2 k, 2}^{*}\left(x_{1}(t-d)\right) \\
& +\frac{1}{l_{1}} \xi_{1 k}^{2} \Psi_{2 k, 2}\left(x_{1}\right)+\xi_{2 k}^{2}(t-d) \Psi_{2 k, 1}^{*}\left(\bar{x}_{2}(t-d)\right),
\end{aligned}
$$

$$
\begin{aligned}
\frac{2}{l_{1}}\left|\frac{\partial W_{2 k}}{\partial x_{1}} \dot{x}_{1}\right|+\frac{1}{l_{1} l_{2}} \xi_{1 k} \dot{x}_{1} \leq & \xi_{2 k}^{2} \Phi_{2 k, 1}\left(\bar{x}_{2}\right)+\frac{1}{l_{1}} \xi_{1 k}^{2} \Phi_{2 k, 2}\left(x_{1}\right) \\
& +\frac{1}{l_{1}} \xi_{1 k}^{2}(t-d) \Phi_{2 k}^{*}\left(x_{1}(t-d)\right),
\end{aligned}
$$

where $\Psi_{2 k, j}, \Psi_{2 k, j}^{*}, \Phi_{2 k, j}, j=1,2$, and $\Phi_{2 k}^{*}$ are nonnegative $\mathscr{C}^{\infty}$ functions for $\forall k \in M$.

Constructing the Lyapunov-Krasovskii functional

$$
\begin{aligned}
V_{2 k}= & \widetilde{V}_{2 k}+\int_{t-d}^{t} \xi_{2 k}^{2}(\mu) \Psi_{2 k, 1}^{*}\left(\bar{x}_{2}(\mu)\right) \mathrm{d} \mu \\
& +\int_{t-d}^{t} \frac{1}{l_{1}(\mu)} \xi_{1 k}^{2}(\mu)\left(\Psi_{2 k, 2}^{*}\left(x_{1}(\mu)\right)+\Phi_{2 k}^{*}\left(x_{1}(\mu)\right)\right) \mathrm{d} \mu,
\end{aligned}
$$

which together with (16) and (17) yields

$$
\begin{aligned}
\dot{V}_{2 k} \leq & -\left(1+\alpha_{1 k}\left(x_{1}\right)\right) \xi_{1 k}^{2}-\frac{n-1}{2} \xi_{1 k}^{2}-\frac{i_{1}}{2 l_{1}^{2}} \xi_{1 k}^{2} \\
& +\frac{1}{l_{1}} \xi_{1 k}^{2}\left(\Psi_{2 k, 2}\left(x_{1}\right)+\Psi_{2 k, 2}^{*}\left(x_{1}\right)+\Phi_{2 k, 2}\left(x_{1}\right)+\Phi_{2 k}^{*}\left(x_{1}\right)\right) \\
& +\left(\frac{1}{l_{1}(t)}-\frac{1}{l_{1}(t-d)}\right) \xi_{1 k}^{2}(t-d) \\
& \times\left(\Psi_{2 k, 2}^{*}\left(x_{1}(t-d)\right)+\Phi_{2 k}^{*}\left(x_{1}(t-d)\right)\right) \\
& \left.+\frac{1}{l_{1}}\left(1+\frac{1}{l_{2}}\right) \xi_{2 k}^{2-\left(1 / p_{1 k}\right.}\right)\left(x_{3 k}^{* p_{2 k}}+x_{3}^{p_{2 k}}-x_{3 k}^{* p_{2 k}}\right) \\
& +\xi_{2 k}^{2}\left(3+\Psi_{2 k, 1}\left(\bar{x}_{2}\right)+\Psi_{2 k, 1}^{*}\left(\bar{x}_{2}\right)+\Phi_{2 k, 1}\left(\bar{x}_{2}\right)\right) \\
& -\frac{i_{1}}{l_{1}^{2}} W_{2 k}-\frac{i_{1} l_{2}+l_{1} \dot{l}_{2}}{l_{1}^{2} l_{2}^{2}}\left(W_{2 k}+\frac{\xi_{1 k}^{2}}{2}\right) .
\end{aligned}
$$

To guarantee the boundedness of $l_{1}$, we design the following gain update law as

$$
i_{1}=\max \left\{-l_{1}^{2}+2 l_{1} \eta_{1 k}\left(x_{1}\right), 0\right\}, \quad l_{1}(0)=1,
$$

where $\quad \eta_{1 k}\left(x_{1}\right)=\Psi_{2 k, 2}\left(x_{1}\right)+\Psi_{2 k, 2}^{*}\left(x_{1}\right)+\Phi_{2 k, 2}\left(x_{1}\right)+\Phi_{2 k}^{*}$ $\left(x_{1}\right), k \in M$.

From (21), it can be concluded that 


$$
\begin{aligned}
0 & <i_{1} \leq 2 l_{1} \eta_{1 k}\left(x_{1}\right), \\
l_{1} & \geq-l_{1}^{2}+2 l_{1} \eta_{1 k}\left(x_{1}\right), \\
l_{1}(t) & \geq l_{1}(t-d) \geq 1,
\end{aligned}
$$

which leads to

$$
\begin{aligned}
\frac{i_{1}}{2 l_{1}^{2}} \xi_{1 k}^{2} & \leq \frac{1}{2} \xi_{1 k}^{2}-\frac{1}{l_{1}} \xi_{1 k}^{2} \eta_{1 k}\left(x_{1}\right), \\
\frac{1}{l_{1}(t)}-\frac{1}{l_{1}(t-d)} & \leq 0, \\
-\frac{i_{1}}{l_{1}^{2}} W_{2 k}-\frac{i_{1} l_{2}+l_{1} i_{2}}{l_{1}^{2} l_{2}^{2}}\left(W_{2 k}+\frac{\xi_{1 k}^{2}}{2}\right) & \leq-\frac{i_{2}}{l_{1} l_{2}^{2}}\left(W_{2 k}+\frac{\xi_{1 k}^{2}}{2}\right) .
\end{aligned}
$$

Substituting (21) and (23) into (20), it yields

$$
\begin{aligned}
\dot{V}_{2 k} \leq & -\left(1+\alpha_{1 k}\left(x_{1}\right)\right) \xi_{1 k}^{2}-\frac{n-2}{2} \xi_{1 k}^{2} \\
& +\frac{1}{l_{1}}\left(1+\frac{1}{l_{2}}\right) \xi_{2 k}^{2-1 / p_{1 k}} x_{3 k}^{* p_{2 k}}+\xi_{2 k}^{2}\left(3+\Psi_{2 k, 1}\left(\bar{x}_{2}\right)\right. \\
& \left.+\Psi_{2 k, 1}^{*}\left(\bar{x}_{2}\right)+\Phi_{2 k, 1}\left(\bar{x}_{2}\right)\right)-\frac{i_{2}}{l_{1} l_{2}^{2}}\left(W_{2 k}+\frac{\xi_{1 k}^{2}}{2}\right) \\
& +\frac{1}{l_{1}}\left(1+\frac{1}{l_{2}}\right) \xi_{2 k}^{2-1 / p_{1 k}}\left(x_{3}^{p_{2 k}}-x_{3 k}^{* p_{2 k}}\right) .
\end{aligned}
$$

We design the virtual controller

$$
\begin{aligned}
x_{3 k}^{* p_{2 k}}= & -l_{1} \xi_{2 k}^{\left(1 / p_{1 k}\right)}\left(n+3+\alpha_{2 k}\left(\bar{x}_{2}\right)+\Psi_{2 k, 1}\left(\bar{x}_{2}\right)\right. \\
& \left.+\Psi_{2 k, 1}^{*}\left(\bar{x}_{2}\right)+\Phi_{2 k, 1}\left(\bar{x}_{2}\right)\right) \\
\triangleq & -l_{1}\left(\xi_{2 k} \beta_{2 k}\left(\bar{x}_{2}\right)\right)^{\left(1 / p_{1 k}\right)} .
\end{aligned}
$$

By Lemmas 1 and 2, one has

$$
2\left|\xi_{2 k}^{2-1 / p_{1 k}}\left(x_{3}^{p_{2 k}}-x_{2}^{* p_{2 k}}\right)\right| \leq \xi_{2 k}^{2}+b_{2} \xi_{2}^{3},
$$

where $\xi_{2}=x_{3}^{p_{1 k} p_{2 k}}-x_{2}^{*} p_{1 k} p_{2 k}$ and $b_{2}>0$ is a constant.

Combining (24)-(26), one has

$$
\begin{aligned}
\dot{V}_{2 k} \leq & -\sum_{j=1}^{2}\left(1+\alpha_{j k}\left(\bar{x}_{j}\right)\right) \xi_{j k}^{2}-(n-2)\left(\frac{\xi_{1 k}^{2}}{2}+\xi_{2 k}^{2}\right) \\
& +b_{3 k} \xi_{3 k}^{2}-\frac{i_{2}}{l_{1} l_{2}^{2}}\left(W_{2 k}+\frac{\xi_{1 k}^{2}}{2}\right) .
\end{aligned}
$$

Inductive steps: assume at step $i-1$, there is a Lyapunov-Krasovskii functional $V_{i-1, k}, k \in M$, a chain of dynamic gains $l_{j} \geq 1, j=1, \ldots, i-1$, depicted by

$$
\begin{array}{r}
i_{1}=\max \left\{-l_{1}^{2}+2 l_{1} \eta_{1 k}\left(x_{1}\right), 0\right\}, \quad l_{1}(0)=1, \\
i_{j}=\max \left\{-\delta_{j k} l_{j}^{2}+2 l_{j} \eta_{j k}\left(\bar{l}_{j-1}, \bar{x}_{j}\right), 0\right\}, \quad l_{j}(0)=1, \\
(j=2, \ldots, i-2)
\end{array}
$$

where $\quad \delta_{j k}=1 /\left(2^{p_{1 k} \cdots p_{j-1, k}}-1\right), \quad \eta_{j k}(\cdot), \quad j=1, \ldots, i-2$, $k \in M$ are positive $\mathscr{C}^{\infty}$ functions and a series of virtual controllers $x_{1 k}^{*}, \ldots, x_{i k}^{*}$ given by

$$
\begin{aligned}
x_{1 k}^{*} & =0, \quad \xi_{1 k}=x_{1}-x_{1 k}^{*}, \\
x_{2 k}^{*} & =-\xi_{1 k} \beta_{1 k}\left(x_{1}\right), \quad \xi_{2 k}=x_{2}^{p_{1 k}}-x_{2 k}^{* p_{1 k}}, \\
\vdots & \\
x_{i k}^{* p_{1 k} \cdots p_{i-1, k}} & =-\left(l_{1} \cdots l_{i-2}\right)^{p_{1 k} \cdots p_{i-2, k}} \xi_{i-1, k} \beta_{i-1, k}\left(\bar{l}_{i-3}, \bar{x}_{i-1}\right), \\
\xi_{i k} & =x_{i}^{p_{1 k} \cdots p_{i-1, k}}-x_{i k}^{* p_{1 k} \cdots p_{i-1, k},}
\end{aligned}
$$

with $\beta_{j k}(\cdot), j=1, \ldots, i-1, k \in M$ being positive $\mathscr{C}^{\infty}$ functions such that

$$
\begin{aligned}
\dot{V}_{i-1, k} & \leq-\sum_{j=1}^{i-1}\left(1+\alpha_{j k}\left(\bar{x}_{j}\right)\right) \xi_{j k}^{2}+b_{i k} \xi_{i k}^{2} \\
& -(n-i+1)\left(\frac{\xi_{1 k}^{2}}{2}+\sum_{j=2}^{i-1} \xi_{j k}^{2}\right) \\
& -\frac{i_{i-1}}{l_{1} \cdots l_{i-2} l_{i-1}^{2}}\left(W_{2 k}\left(\bar{x}_{2}\right)+\cdots+W_{i-1, k}\left(\bar{l}_{i-3}, \bar{x}_{i-1}\right)+\frac{\xi_{1 k}^{2}}{2}\right),
\end{aligned}
$$

where $\alpha_{j k}(\cdot) j=1, \ldots, i-1$ are $\mathscr{C}^{\infty}$ functions and $b_{i k}>0$.

In the following, we will show that (30) is also held at step $i$. With this regard, consider the following Lyapunov-Krasovskii functional as

$$
\begin{aligned}
\tilde{V}_{i k}= & V_{i-1, k}+\frac{1}{l_{1} \cdots l_{i-1}}\left(1+\frac{1}{l_{i}}\right) W_{i k}\left(\bar{l}_{i-1}, \bar{x}_{i}\right) \\
& +\frac{1}{l_{1} \cdots l_{i}}\left(\sum_{j=2}^{i-1} W_{j k}\left(\bar{l}_{j-2}, \bar{x}_{j}\right)+\frac{1}{2} \xi_{1 k}^{2}\right),
\end{aligned}
$$

where $l_{i} \geq 1$ is a dynamic gain to be determined later and $W_{i k}=\int_{x_{i k}^{*}}^{x_{i}}\left(\mu^{p_{1 k} \cdots p_{i-1, k}}-x_{i k}^{* p_{1 k} \cdots p_{i-1, k}}\right)^{2-1 /\left(p_{1 k} \cdots p_{i-1, k}\right)} \mathrm{d} \mu$.

Following the same line, we can obtain that $W_{i k}\left(\bar{l}_{i-2}, \bar{x}_{i}\right)$ is $\mathscr{C}^{1}$, and

$$
\begin{aligned}
\frac{\partial W_{i k}}{\partial x_{i}}= & \xi_{i k}^{2-\left(1 / p_{1 k} \cdots p_{i-1, k}\right)}, \\
\frac{\partial W_{i k}}{\partial x_{j}}= & \left(\frac{1}{p_{1 k} \cdots p_{i-1, k}}-2\right) \frac{\partial x_{i k}^{*} p_{1 k \cdots} \cdots p_{i-1, k}}{\partial x_{j}} \\
& \times \int_{x_{i k}^{*}}^{x_{i}}\left(\mu^{p_{1 k} \cdots p_{i-1, k}}-x_{i k}^{* p_{1 k} \cdots p_{i-1, k}}\right)^{1-\left(1 / p_{1 k} \cdots p_{i-1, k}\right)} \mathrm{d} \mu, \\
\frac{\partial W_{i k}}{\partial l_{j}}= & \left(\frac{1}{p_{1 k} \cdots p_{i-1, k}}-2\right) \frac{\partial x_{i k}^{*} p_{1 k} \cdots p i-1, k}{\partial l_{j}} \\
& \times \int_{x_{i k}^{*}}^{x_{i}}\left(\mu^{p_{1 k} \cdots p_{i-1, k}}-x_{i k}^{* p_{1 k} \cdots p_{i-1, k}}\right)^{1-\left(1 / p_{1 k} \cdots p_{i-1, k}\right)} \mathrm{d} \mu, \\
\lambda_{i k}\left(x_{i}-x_{i k}^{*}\right)^{2 p_{1 k} \cdots p_{i-1, k}} \leq & W_{i k}\left(\bar{l}_{i-2}, \bar{x}_{i}\right) \\
\leq & \left(2^{p_{1 k} \cdots p_{i-1, k}}-1\right) \xi_{i k}^{2},
\end{aligned}
$$

where $\lambda_{i k}>0$ is a constant.

Due to $l_{j} \geq 1$, and (30)-(32), one has 
Mathematical Problems in Engineering

5

$$
\begin{aligned}
& \dot{\tilde{V}}_{i k} \leq \sum_{j=1}^{i-1}\left(1+\alpha_{j k}\left(\bar{x}_{j}\right)\right) \xi_{j k}^{2}-(n-i+1)\left(\frac{\xi_{1 k}^{2}}{2}+\sum_{j=2}^{i-1} \xi_{j k}^{2}\right) \\
& +b_{i k} \xi_{i k}^{2}-\frac{i_{i-1}}{l_{1} \cdots l_{i-2} l_{i-1}^{2}}\left(\sum_{j=2}^{i-1} W_{j k}+\frac{\xi_{1 k}^{2}}{2}\right) \\
& +\frac{1+l_{i}}{l_{1} \cdots l_{i}}\left(\xi_{i k}^{2-1 /\left(p_{1 k} \cdots p_{i-1, k}\right)}\left(x_{i+1, k}^{* p_{i k}}+x_{i+1}^{p_{i k}}-x_{i+1, k}^{* p_{i k}}\right)\right) \\
& +\frac{2}{l_{1} \cdots l_{i-1}} \mid \xi_{i k}^{2-1 /\left(p_{1 k} \cdots p_{i-1, k}\right)} f_{i k}+\sum_{j=1}^{i-1} \frac{\partial W_{i k}}{\partial x_{j}} \dot{x}_{j} \\
& +\sum_{j=1}^{i-2} \frac{\partial W_{i k}}{\partial l_{j}} i_{j} \mid+\frac{1}{l_{1} \cdots l_{i}}\left(\sum_{j=2}^{i-1}\left(\sum_{p=1}^{j} \frac{\partial W_{j k}}{\partial x_{p}} \dot{x}_{p}+\sum_{p=1}^{j-2} \frac{\partial W_{j k}}{\partial l_{p}} l_{p}\right)+\xi_{1 k} \dot{x}_{1}\right)-\sum_{p=1}^{i-1} \frac{l_{1} \cdots \dot{l}_{p} \cdots l_{i-1}}{l_{1}^{2} \cdots l_{i-1}^{2}} W_{i k} \\
& -\sum_{p=1}^{i} \frac{l_{1} \cdots i_{p} \cdots l_{i}}{l_{1}^{2} \cdots l_{i}^{2}}\left(\sum_{j=1}^{i} W_{j k}+\frac{\xi_{1 k}^{2}}{2}\right)
\end{aligned}
$$

Similarly, we can obtain the following inequalities

$$
\begin{aligned}
& \frac{2}{l_{1} \cdots l_{i-1}}\left|\xi_{i k}^{2-1 /\left(p_{1 k} \cdots p_{i-1, k}\right)} f_{i k}\right| \leq \xi_{i k}^{2} \Psi_{i k, 1}\left(\bar{l}_{i-2}, \bar{x}_{i}\right)+\xi_{i k}^{2}(t-d) \times \Psi_{i k, 1}^{*}\left(\bar{l}_{i-2}(t-d), \bar{x}_{i}(t-d)\right) \\
& +\frac{1}{l_{1} \cdots l_{i-1}}\left(\xi_{1 k}^{2}+\sum_{j=2}^{i-1}\left(x_{j}-x_{j k}^{*}\right)^{2 p_{1 k} \cdots p_{j-1, k}}\right) \times \Psi_{i k, 2}\left(\bar{l}_{i-2}, \bar{x}_{i-1}\right)+\frac{1}{l_{1} \cdots l_{i-1}}\left(\xi_{1 k}^{2}(t-d)\right. \\
& \left.+\sum_{j=2}^{i-1}\left(x_{j}(t-d)-x_{j k}^{*}(t-d)\right)^{2 p_{1 k} \cdots p_{j-1, k}}\right) \times \Psi_{i k, 2}^{*}\left(\bar{l}_{i-2}(t-d), \bar{x}_{i-1}(t-d)\right), \\
& \frac{2}{l_{1} \cdots l_{i-1}}\left|\sum_{j=1}^{i-1} \frac{\partial W_{i k}}{\partial x_{j}} \dot{x}_{j}+\sum_{j=1}^{i-2} \frac{\partial W_{i k}}{\partial l_{j}} \dot{l}_{j}\right| \leq \xi_{i k}^{2} \Phi_{i k, 1}\left(\bar{l}_{i-2}, \bar{x}_{i}\right)+\xi_{i k}^{2}(t-d) \times \Phi_{i k, 1}^{*}\left(\bar{l}_{i-2}(t-d), \bar{x}_{i}(t-d)\right) \\
& +\frac{1}{l_{1} \cdots l_{i-1}}\left(\xi_{1 k}^{2}+\sum_{j=2}^{i-1}\left(x_{j}-x_{j k}^{*}\right)^{2 p_{1 k} \cdots p_{j-1, k}}\right) \times \Phi_{i k, 2}\left(\bar{l}_{i-2}, \bar{x}_{i-1}\right) \\
& +\frac{1}{l_{1} \cdots l_{i-1}}\left(\xi_{1 k}^{2}(t-d)+\sum_{j=2}^{i-1}\left(x_{j}(t-d)-x_{j k}^{*}(t-d)\right)^{2 p_{1 k} \cdots p_{j-1, k}}\right) \times \Phi_{i k, 2}^{*}\left(\bar{l}_{i-2}(t-d), \bar{x}_{i-1}(t-d)\right), \\
& \frac{1}{l_{1} \cdots l_{i}}\left(\sum_{j=2}^{i-1}\left(\sum_{p=1}^{j} \frac{\partial W_{j k}}{\partial x_{p}} \dot{x}_{p}+\sum_{p=1}^{j-2} \frac{\partial W_{j k}}{\partial l_{p}} \dot{l}_{p}\right)+\xi_{1 k} \dot{x}_{1}\right) \leq \frac{\Omega_{i k}\left(\bar{l}_{i-2}, \bar{x}_{i-1}\right)}{l_{1} \cdots l_{i-1}}\left(\xi_{1 k}^{2}+\sum_{j=2}^{i-1}\left(x_{j}-x_{j k}^{*}\right)^{2 p_{1 k} \cdots p_{j-1, k}}\right) \\
& +\xi_{i k}^{2}+\frac{1}{l_{1} \cdots l_{i-1}} \Omega_{i k}^{*}\left(\bar{l}_{i-2}(t-d), \bar{x}_{i-1}(t-d)\right) \times\left(\xi_{1 k}^{2}(t-d)+\sum_{j=2}^{i-1}\left(x_{j}(t-d)-x_{j k}^{*}(t-d)\right)^{2 p_{1 k} \cdots p_{j-1, k}}\right),
\end{aligned}
$$


where $\Psi_{i k, j}, \Psi_{i k, j}^{*}, \Phi_{i k, j}, \Phi_{i k, j}^{*}, \Omega_{i k}$, and $\Omega_{i k}^{*}, j=1,2, k \in M$, are nonnegative $\mathscr{C}^{\infty}$ functions.
Choose the Lyapunov-Krasovskii functional

$$
\begin{aligned}
V_{i k}= & \widetilde{V}_{i k}+\int_{t-d}^{t} \xi_{i k}^{2}(\mu)\left(\Psi_{i k, 1}^{*}\left(\bar{l}_{i-2}(\mu), \bar{x}_{i}(\mu)\right)+\Phi_{i k, 1}^{*}\left(\bar{l}_{i-2}(\mu) s, \bar{x}_{i}(\mu)\right)\right) \mathrm{d} \mu \\
& +\int_{t-d}^{t} \frac{1}{l_{1}(\mu) \cdots l_{i-1}(\mu)} \times\left(\xi_{1 k}^{2}(\mu)+\sum_{j=2}^{i-1}\left(x_{j}(\mu)-x_{j k}^{*}(\mu)\right)^{2 p_{1 k} \cdots p_{j-1, k}}\right) \\
& \times\left(\Psi_{i k, 2}^{*}\left(\bar{l}_{i-2}(\mu), \bar{x}_{i-1}(\mu)\right)+\Phi_{i k, 2}^{*}\left(\bar{l}_{i-2}(\mu), \bar{x}_{i-1}(\mu)\right)\right. \\
& \left.+\Omega_{i k}^{*}\left(\bar{l}_{i-2}(\mu), \bar{x}_{i-1}(\mu)\right)\right) \mathrm{d} \mu .
\end{aligned}
$$

The application of (33)-(37) gives rise to

$$
\begin{aligned}
\dot{V}_{i k} \leq & \sum_{j=1}^{i-1}\left(1+\alpha_{j k}\left(\bar{x}_{j}\right)\right) \xi_{j k}^{2}+b_{i k} \xi_{i k}^{2} \\
& -(n-i+1)\left(\frac{\xi_{1 k}^{2}}{2}+\sum_{j=2}^{i-1} \xi_{j k}^{2}\right)-\frac{\dot{l}_{i-1}}{l_{1} \cdots l_{i-2} l_{i-1}^{2}}\left(\sum_{j=2}^{i-1} W_{j k}+\frac{\xi_{1 k}^{2}}{2}\right) \\
& +\frac{1}{l_{1} \cdots l_{i-1}}\left(\xi_{1 k}^{2}+\sum_{j=2}^{i-1}\left(x_{j}-x_{j k}^{*}\right)^{2 p_{1 k} \cdots p_{j-1, k}}\right) \times\left(\Psi_{i k, 2}\left(\bar{l}_{i-2}, \bar{x}_{i-1}\right)+\Psi_{i k, 2}^{*}\left(\bar{l}_{i-2}, \bar{x}_{i-1}\right)+\Phi_{i k, 2}\left(\bar{l}_{i-2}, \bar{x}_{i-1}\right)\right. \\
& \left.+\Phi_{i k, 2}^{*}\left(\bar{l}_{i-2}, \bar{x}_{i-1}\right)+\Omega_{i k}\left(\bar{l}_{i-2}, \bar{x}_{i-1}\right)+\Omega_{i k}^{*}\left(\bar{l}_{i-2}, \bar{x}_{i-1}\right)\right) \\
& +\left(\frac{1}{l_{1} \cdots l_{i-1}}-\frac{1}{l_{1}(t-d) \cdots l_{i-1}(t-d)}\right) \\
& \times\left(\xi_{1 k}^{2}(t-d)+\sum_{j=2}^{i-1}\left(x_{j}(t-d)-x_{j k}^{*}(t-d)\right)^{2 p_{1 k} \cdots p_{j-1, k}}\right) \\
& \times\left(\Psi_{i k, 2}^{*}\left(\bar{l}_{i-2}(t-d), \bar{x}_{i-1}(t-d)\right)+\Phi_{i k, 2}^{*}\left(\bar{l}_{i-2}(t-d), \bar{x}_{i-1}(t-d)\right)\right. \\
& \left.+\Omega_{i k}^{*}\left(\bar{l}_{i-2}(t-d), \bar{x}_{i-1}(t-d)\right)\right)+\frac{1+l_{i}}{l_{1} \cdots l_{i}}\left(\xi_{i k}^{2-1 /\left(p_{1 k} \cdots p_{i-1, k}\right.}\left(x_{i+1, k}^{* p_{i k}}+x_{i+1}^{p_{i k}}-x_{i+1, k}^{*}\right)\right) \\
& +\xi_{i k}^{2}\left(1+\Psi_{i k, 1}\left(\bar{l}_{i-2}, \bar{x}_{i}\right)+\Psi_{i k, 1}^{*}\left(\bar{l}_{i-2}, \bar{x}_{i}\right)+\Phi_{i k, 1}\left(\bar{l}_{i-2}, \bar{x}_{i}\right)+\Phi_{i k, 1}^{*}\left(\bar{l}_{i-2}, \bar{x}_{i}\right)\right) \\
& -\sum_{p=1}^{i-1} \frac{l_{1} \cdots \dot{l}_{p} \cdots l_{i-1}}{l_{1}^{2} \cdots l_{i-1}^{2}} W_{i k}-\sum_{p=1}^{i} \frac{l_{1} \cdots \dot{l}_{p} \cdots l_{i}}{l_{1}^{2} \cdots l_{i}^{2}} \times\left(\sum_{j=1}^{i} W_{j k}+\frac{\xi_{1 k}^{2}}{2}\right) . \\
&
\end{aligned}
$$

Similar to Step 2, we design the gain update law as

$$
i_{i-1}=\max \left\{-\delta_{i-1, k} l_{i-1}^{2}+2 l_{i-1} \eta_{i-1, k}\left(\bar{l}_{i-2}, \bar{x}_{i-1}\right), 0\right\},
$$

where $l_{i-1}(0)=1, \delta_{i-1, k}=1 /\left(2^{p_{1 k} \cdots p_{i-2, k}}-1\right)$ and $\eta_{i-1, k}\left(\bar{l}_{i-2}\right.$, $\left.\bar{x}_{i-1}\right)=c_{i-1, k}\left(\Psi_{i k, 2}+\Psi_{i k, 2}^{*}+\Phi_{i k, 2}+\Phi_{i k, 2}^{*}+\Omega_{i k}+\Omega_{i k}^{*}\right) \quad$ with $c_{i-1, k}=\max \left\{1,\left(1 / 2 \lambda_{2 k}\right), \ldots,\left(1 / 2 \lambda_{i-1, k}\right)\right\}, k \in M$.
By (39), it is easily concluded that

$$
\begin{aligned}
0 & \leq i_{i-1} \leq 2 l_{i-1} \eta_{i-1, k}(\cdot), \\
i_{i-1} & \geq-\delta_{i-1, k} l_{i-1}^{2}+2 l_{i-1} \eta_{i-1, k}(\cdot), \\
l_{i-1}(t) & \geq l_{i-1}(t-d) \geq 1 .
\end{aligned}
$$

Utilizing (32) and (40), it can be verified that 


$$
\begin{aligned}
& -\frac{i_{i-1}}{l_{1} \cdots l_{i-2} l_{i-1}^{2}}\left(\sum_{j=2}^{i-1} W_{j k}+\frac{\xi_{1 k}^{2}}{2}\right) \leq \frac{\xi_{1 k}^{2}}{2}+\sum_{j=2}^{i-1} \xi_{j k}^{2} \\
& -\frac{\eta_{i-1, k}}{2 c_{i-1, k} l_{1} \cdots l_{i-1}} \times\left(\xi_{1 k}^{2}+\sum_{j=2}^{i-1}\left(x_{j}-x_{j k}^{*}\right)^{2 p_{1 k} \cdots p_{j-1, k}}\right), \\
& \frac{1}{l_{1}(t) \cdots l_{i-1}(t)}-\frac{1}{l_{1}(t-d) \cdots l_{i-1}(t-d)} \leq 0 \\
& -\sum_{p=1}^{i-1} \frac{l_{1} \cdots l_{p} \cdots l_{i-1}}{l_{1}^{2} \cdots l_{i-1}^{2}} W_{i k}-\sum_{p=1}^{i} \frac{l_{1} \cdots l_{p} \cdots l_{i}}{l_{1}^{2} \cdots l_{i}^{2}} \\
& \times\left(\sum_{j=1}^{i} W_{j k}+\frac{\xi_{1 k}^{2}}{2}\right) \leq-\frac{i_{i}}{l_{1} \cdots l_{i-1} l_{i}^{2}}\left(\sum_{j=1}^{i} W_{j k}+\frac{\xi_{1 k}^{2}}{2}\right) .
\end{aligned}
$$

Substituting (39) and (41) into (38) yields

$$
\begin{aligned}
\dot{V}_{i k} \leq & -\sum_{j=1}^{i-1}\left(1+\alpha_{j k}\left(\bar{x}_{j}\right)\right) \xi_{j k}^{2}-(n-i)\left(\frac{\xi_{1 k}^{2}}{2}+\sum_{j=2}^{i-1} \xi_{j k}^{2}\right) \\
& +\frac{1+l_{i}}{l_{1} \cdots l_{i}}\left(\xi_{i k}^{2-1 /\left(p_{1 k} \cdots p_{i-1, k}\right)}\left(x_{i+1, k}^{* p_{i k}}+x_{i+1}^{p_{i k}}-x_{i+1, k}^{* p_{i k}}\right)\right) \\
& +\xi_{i k}^{2}\left(1+b_{i k}+\Psi_{i k, 1}+\Psi_{i k, 1}^{*}+\Phi_{i k, 1}+\Phi_{i k, 1}^{*}\right) \\
& -\frac{i_{i}}{l_{1} \cdots l_{i-1} l_{i}^{2}}\left(\sum_{j=1}^{i} W_{j k}+\frac{\xi_{1 k}^{2}}{2}\right) .
\end{aligned}
$$

Then, we choose the virtual controller

$$
\begin{aligned}
x_{i+1}^{* p_{i k}}= & -l_{1} \cdots l_{i-1} \xi_{i k}^{1 /\left(p_{1 k} \cdots p_{i-1, k}\right)}\left(n-i+3+b_{i k}+\alpha_{i k}\left(\bar{x}_{i}\right)\right. \\
& \left.+\Psi_{i k, 1}+\Psi_{i k, 1}^{*}+\Phi_{i k, 1}+\Phi_{i k, 1}^{*}\right) \\
\triangleq & -l_{1} \cdots l_{i-1}\left(\xi_{i k} \beta_{i k}\left(\bar{l}_{i-2}, \bar{x}_{i}\right)\right)^{1 /\left(p_{1 k} \cdots p_{i-1, k}\right)},
\end{aligned}
$$

where $\alpha_{i k}(\cdot), \beta_{i k}(\cdot)$ are positive $\mathscr{C}^{\infty}$ functions.

By $l_{j} \geq 1, j=1, \ldots, i-1$, and Lemmas $1-2$, one has

$$
\frac{2}{l_{1} \cdots l_{i-1}}\left|\xi_{i k}^{2-\left(1 /\left(p_{1 k} \cdots p_{i-1, k}\right)\right)}\left(x_{i+1}^{p_{i k}}-x_{i+1, k}^{* p_{i k}}\right)\right| \leq \xi_{i k}^{2}+b_{i+1, k} \xi_{i+1, k}^{2} \text {, }
$$

where $\xi_{i+1, k}=x_{i+1}^{p_{1 k} \cdots p_{i k}}-x_{i+1, k}^{* p_{1 k} \cdots p_{i k}}$ and $b_{i+1, k}>0$ is a constant. Substituting (43) and (44) into (42) leads to

$$
\begin{aligned}
\dot{V}_{i k} \leq & \sum_{j=1}^{i}-\left(1+\alpha_{j k}\left(\bar{x}_{j}\right)\right) \xi_{j k}^{2}-(n-i)\left(\frac{\xi_{1 k}^{2}}{2}+\sum_{j=2}^{i-1} \xi_{j k}^{2}\right) \\
& +b_{i+1, k} \xi_{i+1, k}^{2}-\frac{i_{i}}{l_{1} \cdots l_{i-1} l_{1}^{2}}\left(\sum_{j=2}^{i} W_{j k}+\frac{\xi_{1 k}^{2}}{2}\right) .
\end{aligned}
$$

This completes the inductive proof.

Step $n$. During the inductive argument, there exists a dynamic state-feedback controller

$$
\begin{aligned}
u_{k} & =x_{n+1, k}^{*}=-\left(l_{1} \cdots l_{n-1}\right)^{1 / p_{n k}} \xi_{n k}^{1 / p_{1 k} \cdots p_{n k}}\left(3+b_{n k}+\alpha_{n k}(x)+\Psi_{n k, 1}+\Psi_{n k, 1}^{*}+\Phi_{n k, 1}+\Phi_{n k, 1}^{*}\right)^{1 / p_{n k}} \\
& \triangleq-\left(l_{1} \cdots l_{n-1}\right)^{1 / p_{n k}}\left(\xi_{n k} \beta_{n k}\left(\bar{l}_{n-2}, x\right)\right)^{1 / p_{1 k} \cdots p_{n k}},
\end{aligned}
$$

such that

$$
\dot{V}_{n k} \leq-\sum_{j=1}^{n}\left(1+\alpha_{j k}\left(\bar{x}_{j}\right)\right) \xi_{j k}^{2}
$$

\section{Stability Analysis}

For simplicity, let $\widetilde{V}_{k}=\widetilde{V}_{n k}$ and $V_{k}=V_{n k}$. Now, we are ready to present the main result of this paper.

Theorem 1. For switched nonlinear time-delay system (1), there are the following dynamic state-feedback controllers as

$$
\begin{aligned}
\dot{L} & =\eta_{k}(L, x), & & L \in \mathbb{R}^{n-1}, \\
u_{k} & =\beta_{k}(L, x), & & k \in M,
\end{aligned}
$$

where $\eta_{k}: \mathbb{R}^{n-1} \times \mathbb{R}^{n} \longrightarrow \mathbb{R}^{n-1}$ and $\beta_{k}: \mathbb{R}^{n-1} \times \mathbb{R}^{n} \longrightarrow \mathbb{R}$ are continuous functions, and positive $\mathscr{C}^{\infty}$ functions $\alpha_{i k}\left(\bar{x}_{i}\right)$ and $\beta_{i k}\left(\bar{l}_{i-2}, \bar{x}_{i}\right), i=1, \ldots, n, k \in M$, and continuous functions $\gamma_{k p}(x)>0, k, p \in M$ such that

$$
\frac{\partial \widetilde{V}_{k}}{\partial x} G_{k}(x)-\sum_{j=1}^{n} \alpha_{j k}\left(\bar{x}_{j}\right) \xi_{j k}^{2}+\sum_{p=1}^{m} \gamma_{k p}(x)\left(V_{p}-V_{k}\right)<0, \quad \forall x \neq 0,
$$

where $\partial \widetilde{V}_{k} / \partial x=\left(\partial \widetilde{V}_{k} / \partial x_{1}, \ldots, \partial \widetilde{V}_{k} / \partial x_{n}\right)$ and $G_{k}(x)=$ $\left(g_{1 k}(x), \ldots, g_{n-1, k}(x), 0\right)^{T}$, and an appropriate switching law (52) to enable the system state converge to origin while keeping the boundedness of the closed-loop system.

Proof. For system (1), we choose the Lyapunov-Krasovskii functional $V_{k}$ and design the controllers as 


$$
\begin{aligned}
u_{k}= & x_{n+1, k}^{*}=-\left(l_{1} \cdots l_{n-1}\right)^{1 / p_{n k}} \xi_{n k}^{1 / p_{1 k} \cdots p_{n k}}\left(3+b_{n k}+\alpha_{n k}(x)\right. \\
& \left.+\Psi_{n k, 1}+\Psi_{n k, 1}^{*}+\Phi_{n k, 1}+\Phi_{n k, 1}^{*}+\Upsilon_{k}(x)\right)^{1 / p_{n k}} \\
\triangleq & -\left(l_{1} \cdots l_{n-1}\right)^{1 / p_{n k}}\left(\xi_{n k} \beta_{n k}\left(\bar{l}_{n-2}, x\right)\right)^{1 / p_{1 k} \cdots p_{n k}},
\end{aligned}
$$

with $\Upsilon_{k}(x)$ being positive $\mathscr{C}^{\infty}$ function, such that

$$
\dot{V}_{k} \leq \frac{\partial \tilde{V}_{k}}{\partial x} G_{k}(x)-\sum_{j=1}^{n}\left(1+\alpha_{j k}\left(\bar{x}_{j}\right)\right) \xi_{j k}^{2}-\xi_{n k}^{2} \Upsilon(x) .
$$

Next, the switching law is chosen as

$$
\sigma(t)=\arg \min _{k \in M}\left\{V_{k}\right\}
$$

Combining (49), (51), and (52), one has

$$
\dot{V}_{k} \leq-\sum_{j=1}^{n} \xi_{j k}^{2}<0, \quad \forall x \neq 0 .
$$
has

On the contrary, using $\sigma(t)=\arg \min _{k \in M}\left\{V_{k}\right\}$, one

$$
V_{\sigma\left(t_{j-1}\right)}\left(t_{j}\right)=V_{\sigma\left(t_{j}\right)}\left(t_{j}\right)
$$

where $t_{j}$ is the $j$ th switching instant. This together with (53) yields for $\forall t \in\left[t_{j}, t_{j+1}\right)$

$$
\begin{aligned}
V_{\sigma\left(t_{j}\right)}(t) & \leq V_{\sigma\left(t_{j}\right)}\left(t_{j}\right)=V_{\sigma\left(t_{j-1}\right)}\left(t_{j}\right) \leq V_{\sigma\left(t_{j-1}\right)}\left(t_{j-1}\right) \\
& =V_{\sigma\left(t_{j-2}\right)}\left(t_{j-1}\right) \leq \cdots \leq V_{\sigma(0)}(0) .
\end{aligned}
$$

The use of (31) and (32) can estimate the lower bound of $V_{k}$. It can be easily verified that $V_{k} \geq\left(1 / 2 c_{n k}\right)\left(x_{1}^{2}+\right.$ $\left.\left(1 / l_{1}\right)\left(x_{2}-x_{2 k}^{*}\right)^{2 p_{1 k}}+\cdots+\left(1 / l_{1} \cdots l_{n-1}\right)\left(x_{n}-x_{n k}^{*}\right)^{2 p_{1 k} \cdots p_{n-1, k}}\right)$, which together with (55), implies the boundedness of $x_{1}$, $\left(x_{i}-x_{i k}^{*}\right)^{2 p_{i k} \cdots p_{i-1, k}} /\left(l_{1} \cdots l_{i-1}\right), i=2, \ldots, n, k \in M$.

By (21), it can be easily found out that the dynamic gain $l_{1}(t)$ follows a monotone and nondecreasing pattern. Then, $l_{1}(t)$ is shown to be bounded by a contradiction argument. Suppose $\lim _{t \longrightarrow+\infty} l_{1}(t)=+\infty$. By continuity, it is clear that $\eta_{1 k}\left(x_{1}\right)$ is bounded. Hence, there exists a constant $T>0$, such that $-l_{1}^{2}+2 l_{1} \eta_{1 k}\left(x_{1}\right) \leq 0$ on $[T,+\infty)$. This together with (21) implies $i_{1}(t)=0$ on $[T,+\infty)$, which apparently goes against $\lim _{t \longrightarrow+\infty} l_{1}(t)=+\infty$. Thus, $l_{1}(t)$ is bounded. This conversely indicates that $x_{2}-x_{2 k}^{*}$ is bounded, so is $x_{2}$ in light of (29). In a recursive manner, it is relatively easy to verify that $l_{i}(t)$ and $x_{i}$ are bounded based on (28) and (29).

Finally, by (53), it is easily seen that $V_{k}(t)$ is monotonically nonincreasing and bounded below by zero, and hence $\lim _{t \rightarrow+\infty} V_{k}(t)$ exists and is finite. Thus, from (54) we can obtain

$$
\begin{aligned}
\int_{0}^{+\infty} \xi_{i k}^{2}(t) \mathrm{d} t \leq & -\int_{0}^{\infty} \dot{V}_{\sigma(t)}(s) \mathrm{d} s=-\left(\int_{0}^{t_{1}} \dot{V}_{\sigma(0)}(s) \mathrm{d} s+\cdots\right. \\
& \left.+\int_{t_{j-1}}^{t_{j}} \dot{V}_{\sigma\left(t_{j-1}\right)}(s) \mathrm{d} s+\int_{t_{j}}^{+\infty} \dot{V}_{\sigma\left(t_{j}\right)}(s) \mathrm{d} s\right) \\
= & V_{\sigma(0)}(0)-V_{\sigma(0)}\left(t_{1}\right)+\cdots+V_{\sigma\left(t_{j-1}\right)}\left(t_{j-1}\right) \\
& -V_{\sigma\left(t_{j-1}\right)}\left(t_{j}\right)+V_{\sigma\left(t_{j}\right)}\left(t_{j}\right)-\lim _{t \longrightarrow+\infty} V_{\sigma\left(t_{j}\right)}(t) \\
= & V_{\sigma(0)}(0)-\lim _{t \longrightarrow+\infty} V_{\sigma\left(t_{j}\right)}(t) \\
\leq & V_{\sigma(0)}(0) \leq+\infty, \quad \forall t \geq 0 .
\end{aligned}
$$

From the boundedness of $l_{1}, \ldots, l_{n-1}$ and $x_{1}, \ldots, x_{n}$, it can be deduced that $\dot{\xi}_{i k}$ is bounded. Thus, by Barbalat's lemma, it can be concluded that $\lim _{t \longrightarrow+\infty} \xi_{i k}(t)=0$, which together with (4) leads to $\lim _{t \rightarrow+\infty} x(t)=0$.

Remark 1. Condition (49) is weaker than the assumption in $[27,28]$. That is, when $g_{i k}=0, i=1, \ldots, n, d=0$ and $V_{l}=V_{k}, l, k \in M,(1)$ is naturally satisfied. This is described in Theorem 1 in [2]. In addition, the flexible choice of $\alpha_{i k}\left(\bar{x}_{i}\right)$ and $\beta_{i k}\left(\bar{l}_{i-2}, \bar{x}_{i}\right), i=1, \ldots, n, k \in M$, increases the flexibility of (49).

Remark 2. For switched nonlinear time-delay systems (1), the stability analysis based on a common Lyapunov function method breaks down, and we shift to employ multiple Lyapunov functions method to effectively solve global statefeedback control problem. In addition, the construction of coordinate changes and the dynamic gain update law of choice plays an important role in the design of delay-independent controllers of all subsystems.

Remark 3. For nonlinear switched systems without timedelay, the triangular form in $[27,28]$ is a special case of system (1) when $g_{i k}(x)=0, p_{i k}=1$ and $d=0$. For nonlinear switched systems without time-delay, the system in [2] is a special case of nontriangular structure when $d=0$. For nonswitched time-delay nonlinear systems, the system in [25] is restricted to a triangular structure when $g_{i k}(x)=0$, $M=\{1\}$.

Remark 4. When the constant time-delay $d$ becomes the time-varying time-delay $d(t)$, Theorem 1 still holds under the assumption: For each $i=1, \ldots, n, d: \mathbb{R}^{+} \longrightarrow \mathbb{R}$ satisfies $0 \leq d(t) \leq \bar{d}$ and $\dot{d}(t) \leq \tau<1$, where $\bar{d}>0$ is an unknown constant and $\tau>0$ is a constant. Moreover, Theorem 1 can also be extended to the more general switched nonlinear systems with multiple time-delays of the form $\quad \dot{x}_{i}=h_{i \sigma(t)}\left(\bar{x}_{i}\right) x_{i+1}^{p_{i \sigma(t)}}+f_{i \sigma(t)}\left(\bar{x}_{i}, x_{1}\left(t-d_{1}(t)\right), \ldots, x_{i}\right.$ $\left.\left(t-d_{i}(t)\right)\right)+g_{i \sigma(t)}(x), \quad i=1, \ldots, n-1, \dot{x}_{n}=h_{n \sigma(t)}(x)$ $u_{\sigma(t)}^{p_{n \sigma(t)}}+f_{n \sigma(t)}\left(x, x_{1}\left(t-d_{1}(t)\right), \ldots, x\left(t-d_{i}(t)\right)\right), x(s)=\zeta$ $(s), s \in[-d, 0]$, under the assumptions: (i) $h_{i \sigma(t)}$, $i=1, \ldots, n$, are $\mathscr{C}^{1}$ functions and have the same sign. (ii) For each $i=1, \ldots, n, d_{i}: \mathbb{R}^{+} \longrightarrow \mathbb{R}$ satisfies $0 \leq d_{i}(t) \leq \bar{d}$ and 
$\dot{d}_{i}(t) \leq \tau<1$, where $\bar{d}>0$ is an unknown constant and $\tau>0$ is a constant.

\section{An Illustrative Example}

Consider the switched nonlinear time-delay system:

$$
\begin{aligned}
& \dot{x}_{1}=x_{2}^{p_{1 \sigma(t)}}+f_{1 \sigma(t)}\left(x_{1}, x_{1}(t-d)\right)+g_{1 \sigma(t)}\left(x_{1}, x_{2}\right), \\
& \dot{x}_{2}=u_{\sigma(t)}^{p_{2 \sigma(t)}}+f_{2 \sigma(t)}(x, x(t-d)),
\end{aligned}
$$

where $x=\left(x_{1}, x_{2}\right)^{T}, \sigma:[0, \infty) \longrightarrow\{1,2\}, p_{11}=1, p_{21}=5$, $f_{11}=x_{1} \sin x_{1}(t-d), g_{11}=x_{1} x_{2}+3 x_{2}, f_{21}=x_{1}^{2} x_{2}(t-d)$ $\sin x_{1}, p_{12}=3, p_{22}=1, f_{12}=x_{1}, g_{12}=2 x_{1} x_{2}+5 x_{2}-x_{1}^{2} x_{2}$, $f_{22}=(1 / 2) x_{2}^{5}(t-d)$.

Clearly, none of the subsystems of (57), even if $d=0$, is stabilizable by any smooth feedback because their linearization at the origin follows a mode that is uncontrollable and relevant to a positive eigenvalue. For this reason, it can be inferred that when $d>0$, there is a need to control the delayinvolved switched system (57) using the nonsmooth feedback. By Theorem 1, a delay-independent dynamic statefeedback controller can be constructed of the following form as $i_{1}=\max \left\{-l_{1}^{2}+2 l_{1} \eta_{1 k}\left(x_{1}\right), 0\right\}, l_{1}(0)=1, u_{k}=-\left(l_{1}^{1 / p_{2 k}}\left(\xi_{2 k}\right.\right.$ $\left.\beta_{2 k}(x)\right)^{1 / p_{1 k} p_{2 k}}, k \in\{1,2\}$, with $\eta_{1 k}\left(x_{1}\right)$ and $\beta_{2 k}$ being smooth functions to be determined. For simulation, let $\xi_{1 k}=x_{1}$, $\xi_{2 k}=x_{2}^{p_{1 k}}+\xi_{1 k} \beta_{1 k}\left(x_{1}\right)$, and

$$
\begin{aligned}
V_{k}= & \frac{1}{2}\left(1+\frac{1}{l_{1}}\right) \xi_{1 k}^{2} \\
& +\int_{t-d}^{t}\left(-\xi_{1 k} \beta_{1 k}\left(x_{1}\right)\right)^{1 / p_{1 k}}\left(\mu^{p_{1 k}}+\xi_{1 k} \beta_{1 k}\left(x_{1}\right)\right)^{2-\left(1 / p_{1 k}\right)} \mathrm{d} \mu \\
& +\int_{t-d}^{t} \xi_{2 k}^{2}(\mu) \Psi_{2 k, 1}^{*}\left(\bar{x}_{2}(\mu)\right) \mathrm{d} \mu \\
& +\int_{t-d}^{t} \frac{1}{l_{1}(\mu)} \xi_{1 k}^{2}(\mu)\left(\Psi_{2 k, 2}^{*}\left(x_{1}(\mu)\right)+\Upsilon_{2 k}^{*}\left(x_{1}(\mu)\right)\right) \mathrm{d} \mu .
\end{aligned}
$$

The switching law is chosen as $\sigma(t)=\operatorname{argmin}_{k \in M}\left\{V_{k}\right\}$. It is clear that the choice of $\eta_{11}\left(x_{1}\right)=9 x_{1}^{2}+30, \eta_{12}\left(x_{1}\right)=$ $9 x_{1}^{4 / 3}+60, \beta_{1 k}=9 / 2, \beta_{21}=\left(1 / l_{1}\right) x_{1}^{2}+35, \beta_{22}=\left(1 / l_{1}\right)\left(x_{2 d}^{3}+\right.$ $\left.(9 / 2) x_{1}\right)^{4 / 3}+64+6\left(x_{2}^{3}+(9 / 2) x_{1}\right)^{4 / 3}, \Psi_{21,1}^{*}=\left(1 / l_{1}\right) x_{1}^{2}, \Psi_{22,1}^{*}$ $=\left(1 / l_{1}\right)\left(x_{2 d}^{3}+(9 / 2) x_{1}\right)^{4 / 3}, \Psi_{2 k, 2}^{*}=0, \Upsilon_{2 k}^{*}=0.5$ renders

$$
\dot{V}_{k} \leq-\sum_{j=1}^{n} \xi_{j k}^{2}<0, \quad \forall x \neq 0 .
$$

As proved in Theorem 1, the signals of the closed-loop system can be bounded in the manner of $x_{1} \longrightarrow l_{1} \longrightarrow x_{2}$ and $\lim _{t \longrightarrow+\infty} x(t)=0$. Figures 1-4 illustrate the reliability of the proposed control approach when $d=|\sin t|$. Figures 5-8 illustrate the reliability of the proposed control approach when $d=0.1$. Through comparison, it can be found that whether the delay is time-varying or constant does not affect the stability of the system, but will affect the stability time.

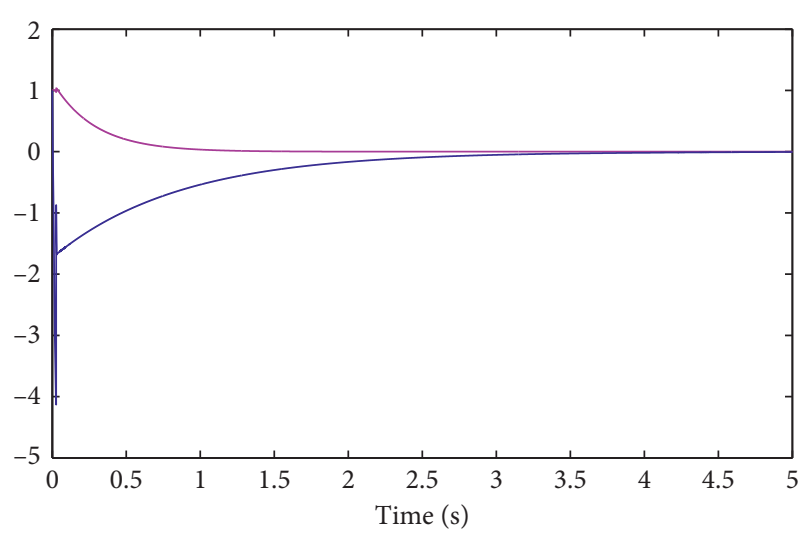

$-x_{1}$

$x_{2}$

Figure 1: Trajectories of $x_{1}$ and $x_{2}$ with $d=|\sin t|$.

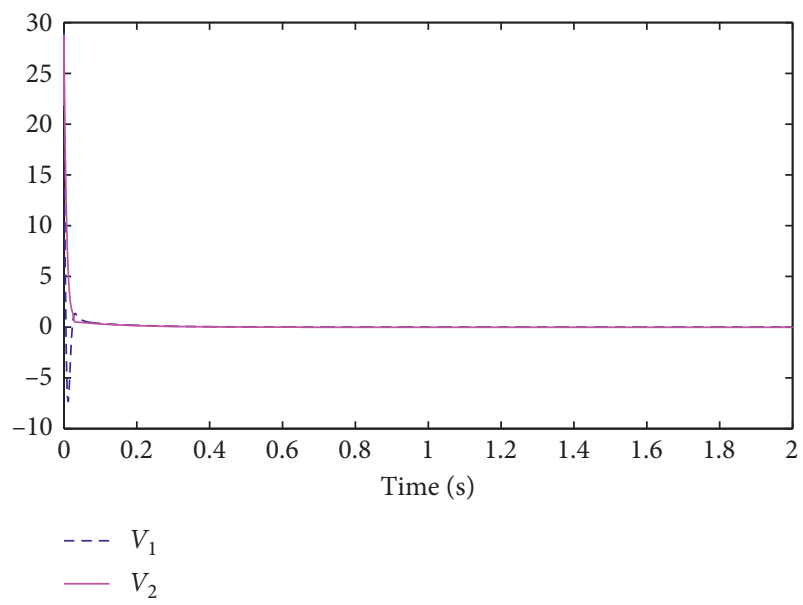

Figure 2: Trajectories of $V_{1}$ and $V_{2}$ with $d=|\sin t|$.

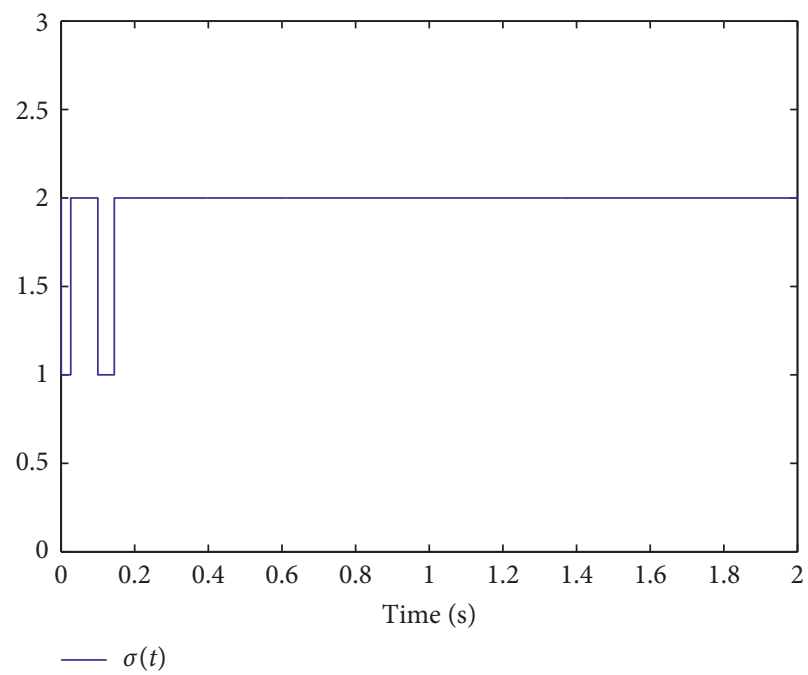

FIgure 3: System switching signal $\sigma(t)$ with $d=|\sin t|$. 


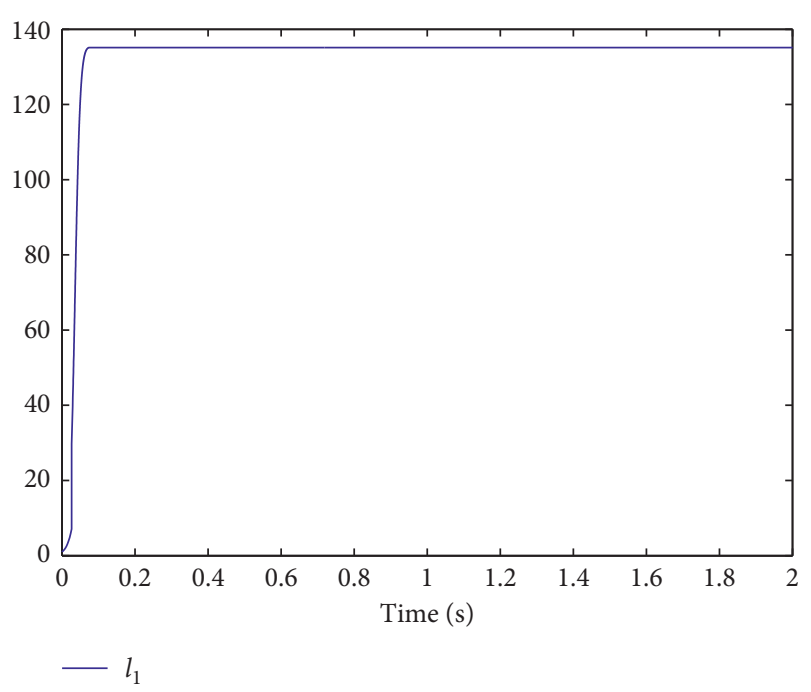

Figure 4: Dynamic gain $l_{1}$ with $d=|\sin t|$.

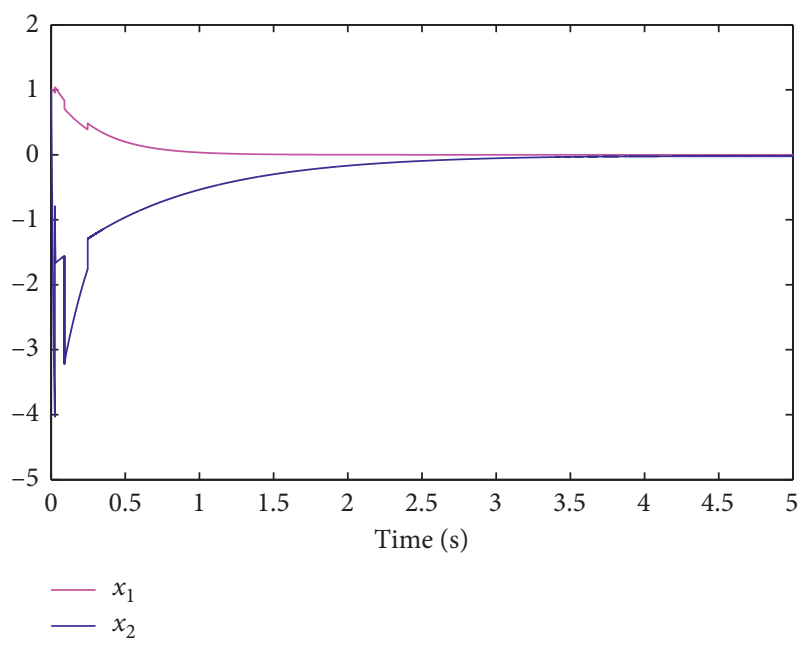

Figure 5: Trajectories of $x_{1}$ and $x_{2}$ with $d=0.1$.

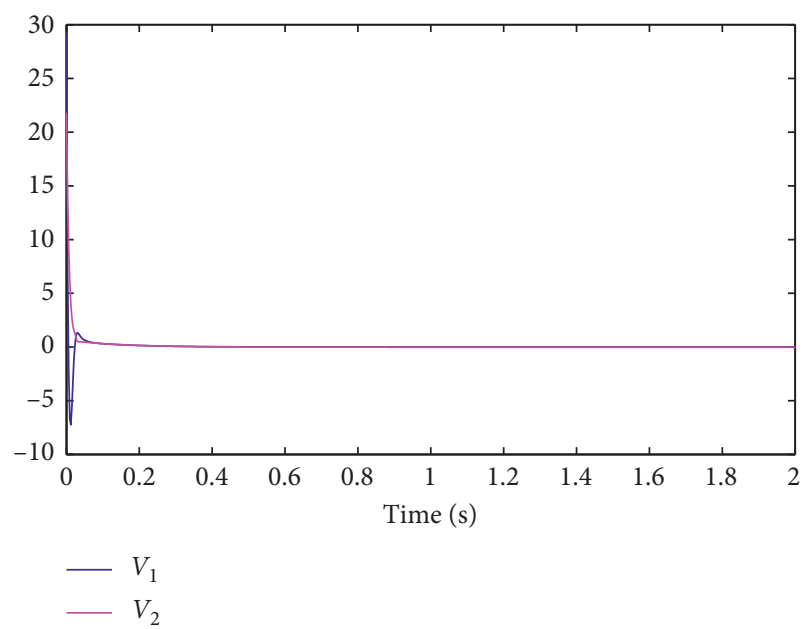

Figure 6: Trajectories of $V_{1}$ and $V_{2}$ with $d=0.1$.

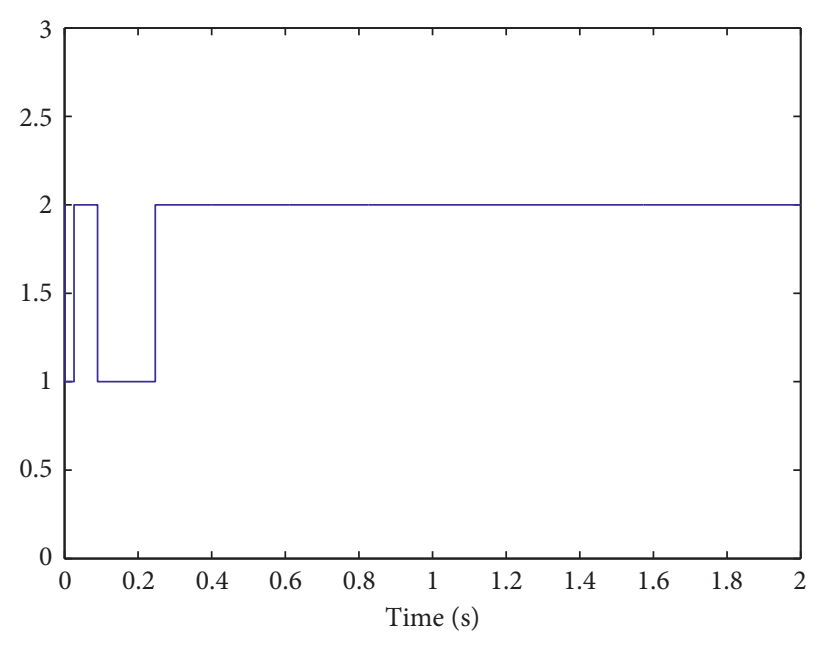

FIGURE 7: System switching signal $\sigma(t)$ with $d=0.1$.

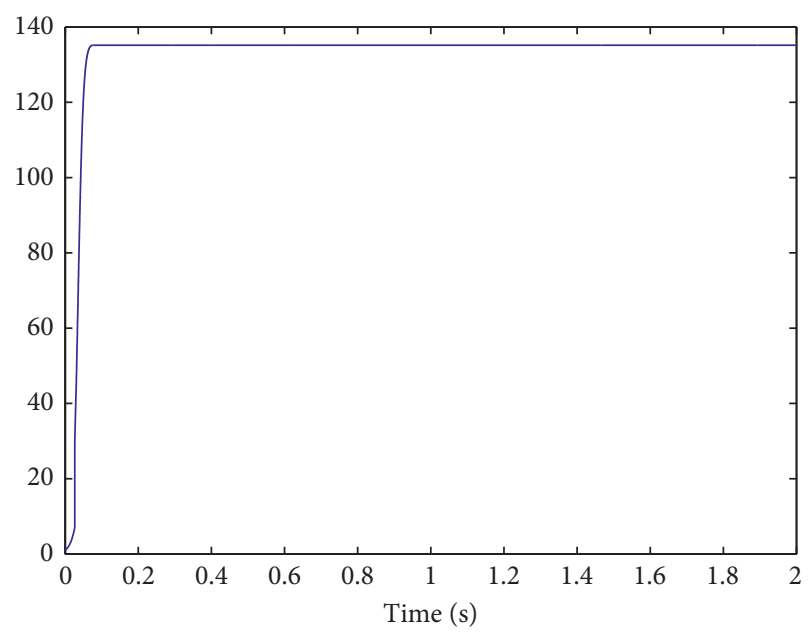

$-l_{1}$

Figure 8: Dynamic gain $l_{1}$ with $d=0.1$.

\section{Conclusion}

This paper discussed the global state-feedback control problem for a class of switched nonlinear time-delay systems. This issue can be solved by using explicitly-constructed coordinate transformations and dynamic gains. The designed controllers can be guaranteed that all signals of the system are bounded; meanwhile, the system state globally converges to the origin. In the future, we will further investigate the problem of adaptive output feedback control for switched nonlinear time-delay systems under weaker conditions. In addition, inspired by [29, 30], we will further discuss the problem of output feedback control for a class of more general switched nonlinear systems with unknown growth rates.

\section{Data Availability}

In our paper, we only use MATLAB for simulation. Therefore, we can only provide simulation programming 
which can be obtained from the corresponding author upon request.

\section{Conflicts of Interest}

The authors declare that there are no conflicts of interest regarding the publication of this paper.

\section{Acknowledgments}

This work was supported by Natural Science Foundation of China (nos. 61773201 and 61622304), Natural Science Foundation of Jiangsu Province (BK20160035), and Jiangsu Overseas Visiting Scholar Program for University Prominent Young \& Middle-aged Teachers and Presidents.

\section{References}

[1] D. Liberzon and A. Morse, "Basic problems in stability and design of switched systems," IEEE Trans. Control System Magazine, vol. 19, no. 5, pp. 59-70, 1999.

[2] L. Long and J. Zhao, "Global stabilization of switched nonlinear systems in non-triangular form and its application," Journal of the Franklin Institute, vol. 351, no. 2, pp. 1161-1178, 2014.

[3] J.-B. Liu, S. Wang, C. Wang, and S. Hayat, "Further results on computation of topological indices of certain networks," IET Control Theory \& Applications, vol. 11, no. 13, pp. 2065-2071, 2017.

[4] A.-Y. Lu and G.-H. Yang, "Input-to-state stabilizing control for cyber-physical systems with multiple transmission channels under denial of service," IEEE Transactions on Automatic Control, vol. 63, no. 6, pp. 1813-1820, 2018.

[5] J.-B. Liu, J. Cao, A. Alofi, A. AL-Mazrooei, and A. Elaiw, "Applications of Laplacian spectra for $n$-prism networks," Neurocomputing, vol. 198, pp. 69-73, 2016.

[6] J.-Y. Zhai, Z. Song, and H. R. Karimi, "Global finite-time control for a class of switched nonlinear systems with different powers via output feedback," International Journal of Systems Science, vol. 49, no. 13, pp. 2776-2783, 2018.

[7] L. Liu, Y.-J. Liu, and S. Tong, "Fuzzy-based multierror constraint control for switched nonlinear systems and its applications," IEEE Transactions on Fuzzy Systems, vol. 27, no. 8, pp. 1519-1531, 2019.

[8] D. Liberzon and R. Tempo, "Common Lyapunov functions and gradient algorithms," IEEE Transactions on Automatic Control, vol. 49, no. 6, pp. 990-994, 2004.

[9] J. Davila, H. Ríos, and L. Fridman, "State observation for nonlinear switched systems using nonhomogeneous highorder sliding mode observers," Asian Journal of Control, vol. 14, no. 4, pp. 911-923, 2012.

[10] L. Liu, Y.-J. Liu, and S. Tong, "Neural networks-based adaptive finite-time fault-tolerant control for a class of strictfeedback switched nonlinear systems," IEEE Transactions on Cybernetics, vol. 49, no. 7, pp. 2536-2545, 2019.

[11] D. Yang, X. Li, and J. Qiu, "Output tracking control of delayed switched systems via state-dependent switching and dynamic output feedback," Nonlinear Analysis: Hybrid Systems, vol. 32, pp. 294-305, 2019.

[12] J.-Y. Zhai, Z.-B. Song, S.-M. Fei, and Z. Zhu, "Global finitetime output feedback stabilisation for a class of switched highorder nonlinear systems," International Journal of Control, vol. 91, no. 1, pp. 170-180, 2018.
[13] X. Yang, X. Li, Q. Xi, and P. Duan, "Review of stability and stabilization for impulsive delayed systems," Mathematical Biosciences \& Engineering, vol. 15, no. 6, pp. 1495-1515, 2018.

[14] H. Ren, G. Zong, and T. Li, "Event-triggered finite-time control for networked switched linear systems with asynchronous switching," IEEE Transactions on Systems, Man, and Cybernetics: Systems, vol. 48, no. 11, pp. 1874-1884, 2018.

[15] L. Liu, Y. Liu, D. Li, S. Tong, and Z. Wang, "Barrier Lyapunov function based adaptive fuzzy FTC for switched systems and its applications to resistance inductance capacitance circuit system," IEEE Trans. Cybernetics, 2019, In press.

[16] G. Zong and H. Ren, "Guaranteed cost finite-time control for semi-Markov jump systems with event-triggered scheme and quantization input," International Journal of Robust and Nonlinear Control, vol. 29, no. 12, pp. 5251-5273, 2019.

[17] D. Liberzon, Switching in Systems and Control, Birkhauser, Boston, MA, USA, 2003.

[18] A. Bacciotti and L. Rosier, Lyipunov Functions and Stability in Control Theory, Springer, New York, NY, USA, 2005.

[19] K. Gu, J. Chen, and V. Kharitonov, Stability of Time-Delay Systems, Springer Science \& Business Media, Berlin, Germany, 2003.

[20] W. Zha, J. Zhai, and S. Fei, "Output feedback control for a class of stochastic high-order nonlinear systems with timevarying delays," International Journal of Robust and Nonlinear Control, vol. 24, no. 12, pp. 2243-2260, 2014.

[21] W. Lin and C. Qian, "Adding one power integrator: a tool for global stabilization of high-order lower-triangular systems," Systems \& Control Letters, vol. 39, no. 5, pp. 339-351, 2000.

[22] X. Zhang, L. Baron, Q. Liu, and E.-K. Boukas, "Design of stabilizing controllers with a dynamic gain for feedforward nonlinear time-delay systems," IEEE Transactions on Automatic Control, vol. 56, no. 3, pp. 692-697, 2011.

[23] X. Zhang and Y. Lin, "Global stabilization of high-order nonlinear time-delay systems by state feedback," Systems \& Control Letters, vol. 65, pp. 89-95, 2014.

[24] J. Zhai and H. R. Karimi, "Universal adaptive control for uncertain nonlinear systems via output feedback," Information Sciences, vol. 500, pp. 140-155, 2019.

[25] X. Zhang, W. Lin, and Y. Lin, "Nonsmooth feedback control of time-delay nonlinear systems: a dynamic gain based approach," IEEE Transactions on Automatic Control, vol. 62, no. 1, pp. 438-444, 2017.

[26] J. Zhai and H. R. Karimi, "Global output feedback control for a class of nonlinear systems with unknown homogenous growth condition," International Journal of Robust and Nonlinear Control, vol. 29, no. 7, pp. 2082-2095, 2019.

[27] J.-L. Wu, "Stabilizing controllers design for switched nonlinear systems in strict-feedback form," Automatica, vol. 45, no. 4, pp. 1092-1096, 2009.

[28] R. Ma and J. Zhao, "Backstepping design for global stabilization of switched nonlinear systems in lower triangular form under arbitrary switchings," Automatica, vol. 46, no. 11, pp. 1819-1823, 2010.

[29] X. Yan, Y. Liu, and W. X. Zheng, "Global adaptive outputfeedback stabilization for a class of uncertain nonlinear systems with unknown growth rate and unknown output function," Automatica, vol. 104, pp. 173-181, 2019.

[30] J. Zhai, "Dynamic output-feedback control for nonlinear time-delay systems and applications to chemical reactor systems," IEEE Transactions on Circuits and Systems II: Express Briefs, vol. 66, no. 11, pp. 1845-1849, 2019. 